\title{
Guidance for practitioners on the use of antiviral drugs to control influenza outbreaks in long-term care facilities in Canada, 2014-2015 season
}

\author{
Fred Y Aoki $M D^{1}$, Upton D Allen MBBS ${ }^{2}, \mathrm{H}$ Grant Stiver $M D^{3}$, Michel Laverdière $M D^{4}$, \\ Danuta Skowronski MD5 ${ }^{5}$, Gerald A Evans $M^{6}$
}

\begin{abstract}
T he purpose of this guidance document is to inform physicians and other health care practitioners of an increased risk of influenza outbreaks in long-term care facilities (LTCF) during the 2014-2015 influenza season in Canada, and to provide recommendations for adjustment to LTCF outbreak control measures on the basis of documented vaccine mismatch.

Influenza seasons dominated by $\mathrm{A}(\mathrm{H} 3 \mathrm{~N} 2)$ viruses are typically associated with greater morbidity and mortality than other types/subtypes of influenza, especially in the elderly (1-3). Significant genetic and antigenic differences have been identified so far in 2014-2015 among circulating $\mathrm{A}(\mathrm{H} 3 \mathrm{~N} 2)$ viruses compared with the 2014-2015 H3N2 vaccine component chosen by the World Health Organization (WHO) for the northern hemisphere $(4,5)$. These differences between circulating viruses and the vaccine strain (called 'antigenic drift' or 'vaccine mismatch') are anticipated to have important implications for reduced vaccine protection this season. Consequently, adjunct protective measures, such as antiviral medications, may require greater emphasis and expanded use in responding to LTCF outbreaks this season.
\end{abstract}

\section{A. PROCESS STATEMENT}

The development of this updated guidance arose in November 2014 from information provided by the Public Health Agency of Canada regarding early data indicating a mismatch of the influenza vaccine $\mathrm{H} 3 \mathrm{~N} 2 \mathrm{com}$ ponent to an emerging H3N2 seasonal strain. This created a need to update recommendations for the use of antiviral drugs for this year's seasonal influenza. The concept was then approved by Association of Medical Microbiology and Infectious Disease (AMMI) Canada. A first draft was cowritten by all the authors (FYA, UDA, HGS, ML, DS and GAE). Subsequently, all the authors reviewed, revised and approved the document before a further review from the AMMI Canada Guidelines Committee and the Infectious Diseases and Immunization Committee of the Canadian Paediatric Society. AMMI Canada approved the final document before submission to the Journal for publication.

\section{B. OVERVIEW OF KEY MESSAGES}

The current guidance document supplements recommendations in the AMMI Canada Guideline 'The use of antiviral drugs for influenza: A foundation document for practitioners' (6). The essential points in this special supplement are: a. Influenza $\mathrm{A}(\mathrm{H} 3 \mathrm{~N} 2)$ viruses are dominating (>90\%) among influenza detections so far in 2014-2015, and have been associated with earlier and more LTCF outbreaks this season compared with usual in several parts of Canada (4). These viruses remain susceptible to the neuraminidase inhibitor (NI) drugs.

b. The majority of influenza $\mathrm{A}(\mathrm{H} 3 \mathrm{~N} 2)$ viruses (>95\%) characterized thus far in Canada this season show genetic and antigenic evidence of mismatch to the vaccine strain (A/Texas/50/2012[H3N2]-like) that had been chosen in February 2014 by the WHO for inclusion in the 2014-2015 trivalent and quadrivalent vaccines for the northern hemisphere $(4,5)$.

c. A substantial number of amino acid (AA) differences between circulating $\mathrm{H} 3 \mathrm{~N} 2$ viruses and the vaccine component are found at key antigenic sites of the hemagglutinin $(\mathrm{H})$ protein of the influenza virus, and number and location of genetic differences are anticipated to substantially affect vaccine effectiveness (VE) this season (7-12).

d. VE estimates against H3N2 for the 2014-2015 season will likely not be available until later in the season (expected in February 2015), but in previous seasons of H3N2 vaccine mismatch, VE estimates of $40 \%$ or lower have been found in association with a comparable or fewer number of antigenic site AA differences between vaccine and $\mathrm{H} 3 \mathrm{~N} 2$ circulating viruses, even in young adults (13-17).

e. In the context of this significant vaccine mismatch, enhanced emphasis and expanded recommendations for antiviral use during LTCF outbreak control are needed while influenza $\mathrm{A}(\mathrm{H} 3 \mathrm{~N} 2)$ activity shows signs of spiking in several parts of Canada.

f. NI drugs, oseltamivir (Tamiflu ${ }^{\circledR}$, Hoffmann-La Roche Ltd, Canada) and zanamivir (Relenza ${ }^{\circledR}$, GlaxoSmithKline Inc, Canada), are the antiviral medications to be prescribed for both treatment and prevention of influenza, including $\mathrm{H} 3 \mathrm{~N} 2$ viruses. Amantadine is not to be prescribed due to resistance of the $\mathrm{H} 3 \mathrm{~N} 2$ virus to its inhibitory action.

g. In view of vaccine mismatch for H3N2 viruses in 2014-2015 and anticipated reduced VE for that component, it is recommended that antiviral chemoprophylaxis be considered for all staff working at the site of a declared influenza $\mathrm{A}(\mathrm{H} 3 \mathrm{~N} 2)$ LTCF outbreak regardless of whether they have received this year's 2014-2015 influenza vaccine.

${ }^{1}$ Professor of Medicine, Medical Microbiology and Pharmacology 87 Therapeutics, Faculty of Medicine, University of Manitoba, Winnipeg,

Manitoba; ${ }^{2}$ Professor, Department of Pediatrics $8 \mathcal{F}$ Institute of Health Policy, Management and Evaluation; Senior Associate Scientist, Research

Institute; Chief, Division of Infectious Diseases, Department of Pediatrics, Hospital for Sick Children, University of Toronto, Toronto, Ontario;

${ }^{3}$ Professor Emeritus of Medicine, Division of Infectious Diseases, Department of Medicine, University of British Columbia, Vancouver, British

Columbia; ${ }^{4}$ Department of Microbiology-Infectious Disease, Hopital Maisonneuve-Rosemont, Montreal, Quebec; ${ }^{5}$ Epidemiology Lead,

Influenza $\mathbb{E}$ Emerging Respiratory Pathogens, BC Centre for Disease Control, Vancouver, British Columbia; ${ }^{6}$ Professor of Medicine,

Biomedical $\mathcal{E}$ Molecular Sciences and Pathology $\mathcal{E}$ Molecular Medicine; Chair, Division of Infectious Diseases, Department of Medicine,

Kingston General Hospital, Queen's University, Kingston, Ontario

Correspondence: Dr Fred Y Aoki, 510-745 Bannatyne Avenue, Winnipeg, Manitoba R3E 0J9. E-mail fred.aoki@umanitoba.ca 


\section{VIROLOGICAL CONTEXT}

1. Dominant influenza $\mathrm{A}(\mathrm{H} 3 \mathrm{~N} 2)$ activity early in the 2014-2015 season

To date during the 2014-2015 season in Canada (current as of week 50, ending December 13, 2014), 4283 (96\%) influenza A viruses and $168(4 \%)$ influenza B viruses have been detected (4). The number of influenza A detections in week 50 nearly doubled from the prior week (week 49, ending December 6, 2014; $n=2354$ ), signifying activity that is beginning to spike. This activity has been driven primarily by increased detection of $\mathrm{A}(\mathrm{H} 3 \mathrm{~N} 2)$ viruses.

Of the 2011 influenza A viruses detected in Canada thus far with subtype information available to December 13, 2014, 2005 (99.7\%) are $\mathrm{A}(\mathrm{H} 3 \mathrm{~N} 2)$ and just six $(0.3 \%)$ are $\mathrm{A}(\mathrm{H} 1 \mathrm{~N} 1) \mathrm{pdm} 09$ (4). This profile is very different from the prior 2013-14 season when $\mathrm{H} 3 \mathrm{~N} 2$ viruses were sparse and $\mathrm{A}(\mathrm{H} 1 \mathrm{~N} 1) \mathrm{pdm} 09$, descended from the 2009 pandemic virus, was instead the dominant influenza A epidemic strain (4).

\section{Evidence for antigenic drift/vaccine mismatch among $\mathrm{H} 3 \mathrm{~N} 2$ viruses detected in Canada}

Of 61 influenza $\mathrm{A}(\mathrm{H} 3 \mathrm{~N} 2)$ viruses characterized so far this season by Canada's influenza reference laboratory, the National Microbiology Laboratory (NML), 59 (97\%) have shown genetic and/or antigenic evidence of antigenic drift and mismatch to the 2014-2015 A/Texas/50/2012(H3N2) vaccine strain (4).

Thirteen $\mathrm{H} 3 \mathrm{~N} 2$ viruses were characterized by standard hemagglutination inhibition (HI) assay and of these, 12 (92\%) met conventional thresholds for titre reduction that define antigenic drift. An additional 58 viruses could not be characterized by $\mathrm{HI}$ and were instead assessed by sequencing of the $\mathrm{H} 3$ gene. Of these, 57 of 58 (98\%) clustered within a genetic group that has been found by $\mathrm{HI}$ assay to be antigenically distinct from the A/Texas/50/2012(H3N2) vaccine strain and, thus, also consistent with antigenic drift in these viruses (4).

\section{Emerging genetic subgroups of $\mathrm{A}(\mathrm{H} 3 \mathrm{~N} 2)$ bear multiple amino acid changes in key antigenic sites with implications for reduced vaccine effectiveness (VE)}

There are several genetic subgroups of H3N2 viruses currently circulating, with nomenclature as described by the European Centre for Disease Prevention and Control (ECDC) in its periodic surveillance reports $(18,19)$. Among 480 global H3 gene sequences collected worldwide between June and November 2014 and publicly available for analysis in the GISAID EpiFlu Database (http://platfrom.gisaid.org), none from the northern or southern hemispheres were found to belong to the same genetic subgroup 3C.1 as the A/Texas/50/2012(H3N2) strain used in the 2014-2015 northern hemisphere vaccine (5) (verbal communication, D Skowronski, BC Centre for Disease Control).

Viruses belonging to phylogenetic subgroups 3C.3, 3C.3a and 3C.2a variously contributed during the summer months (June to August), with lesser contribution of another subgroup that has not yet received separate ECDC designation (provisionally labelled here as 3C.3x). In September 2014, the WHO selected a virus within subgroup 3C.3a, represented by A/Switzerland/9715293/2013(H3N2), to be the vaccine component for the southern hemisphere's upcoming 2015 season (5). These emergent viruses are considered antigenically distinct from the egg-propagated A/Texas/50/2012(H3N2)-like virus (subgroup 3C.1) used in the 2013-2014 and 2014-2015 vaccines for the northern hemisphere $(5,18,19)$.

During the early fall (September to October), subgroup 3C.2a viruses have become more predominant among GISAID sequences collected from the northern hemisphere, and this has been accompanied by lesser contribution from subgroup 3C.3x. This subgroup distribution has also been observed among $\mathrm{H} 3 \mathrm{~N} 2$ viruses collected and sequenced by investigators during the fall 2014 in British Columbia, Canada (verbal communication, D. Skowronski, BC Centre for Disease Control). The emerging subgroups 3C.2a and 3C.3x H3N2 variants each bear multiple amino acid (AA) differences from the $\mathrm{A} /$ Texas/50/2012(H3N2) vaccine strain and from the egg-adapted high-growth reassortant (HGR) provided to manufacturers for vaccine production this season (called X-223A) (5).

Differences between these emerging H3N2 viruses and the 20142015 vaccine strain are found in key antigenic sites of the $\mathrm{H} 3$ protein known to influence antigenicity, immunogenicity and vaccine effectiveness (7-12). In previous seasons, $\mathrm{H} 3 \mathrm{~N} 2$ viruses with comparable or fewer AA substitutions at $\mathrm{H} 3$ antigenic sites relative to vaccine have been associated with VE of approximately $40 \%$ or less in Canada and elsewhere (13-17). One of the particular combinations of H3N2 mutations in subgroup 3C.3x viruses (an asparagine to aspartic acid substitution at position 122 and a leucine to serine substitution at position 157) was associated with VE of $30 \%$ or less in Spain with use of the same A/Texas/50/2012(H3N2) vaccine component in 2013-2014 that has been retained for 2014-2015 (20).

\section{LTCF OUTBREAK CONTROL MEASURES}

LTCF include nursing homes and other institutions that provide health care to individuals, including children, who are unable to manage independently in the community. Some facilities will have multiple smaller units or wards with physical separation and shared- or unit-dedicated staff. These factors will have to be considered in declaring whether a care facility influenza outbreak is facility-wide or specific to a ward/unit.

When influenza virus is causing illness in the community, it may be introduced into the facility by staff, including non-health care workers, newly admitted patients or visitors. Residents of LTCF are at increased risk of severe and/or complicated illness because of frailty, comorbid medical conditions and, in some residents, older age (21). Annual influenza immunization is recommended for these individuals because of these highrisk characteristics, and generally immunization rates are very high among residents. Among health care workers and other staff, influenza immunizations rates are more variable but generally lower than in residents.

Influenza outbreaks among residents are not uncommon in spite of their own high immunization rates. This is probably due, in part, to an impaired immune response associated with aging and other comorbidity. Controlling outbreaks of influenza in LTCF requires a multifaceted approach including:

1. Surveillance for influenza-like illness (ILI)

2. Laboratory testing to identify the cause of ILI

3. Promotion of, and adherence to, infection control guidelines and practices including respiratory etiquette and routine practices, and the use of personal protective equipment

4. Timely communication

5. Influenza immunization for residents and staff

6. Exclusion of ill staff, visitor exclusion and new admission deferral

7. Antiviral drug therapy for ill residents and staff

8. Antiviral drug prophylaxis of non-ill residents and staff

Items 1 through 6 are outside the scope of this guidance and specific recommendations may vary by province. Readers are referred to provincial guidelines and an interim guideline from the United States Centers for Disease Control and Prevention (22).

For control of an outbreak of influenza among residents of a LTCF, surveillance for ILI is critical for early identification and response. FluWatch defines ILI as acute onset of respiratory illness with fever and cough and with one or more of sore throat, arthralgia, myalgia or prostration but further underscores that in patients 65 years and older, fever may not be prominent (4). Where influenza is suspected, laboratory testing can confirm the diagnosis. The preferred sample for individual influenza diagnosis is a nasopharyngeal specimen obtained using a flocked swab. For surveillance purposes and outbreak diagnosis, nasal specimens collected from several patients within a cluster affected by ILI may also be sufficient to determine the cause of the outbreak. However, oropharyngeal secretions obtained with a throat swab are suboptimal for influenza diagnosis. The preferred testing methods are, in order of declining preference: RT-PCR, rapid influenza diagnostic tests and viral culture.

For surveillance purposes, FluWatch defines an influenza outbreak on the basis of two or more cases of ILI within a seven-day period, 
including at least one laboratory-confirmed case of influenza (4). However, this is for surveillance purposes and the declaration of an influenza outbreak and the enactment of control measures is at the discretion of the local health authority/Medical Officer of Health in accordance with provincial guidance. When an influenza outbreak is suspected in a LTCF, this should be reported within $24 \mathrm{~h}$ to the local health authority/Medical Officer of Health to enable timely interventions. It is at the discretion of the local health authority/Medical Officer of Health, in consultation with health care and infection control practitioners, to declare an influenza outbreak in a LTCF and to recommend specific interventions for its control according to provincial guidelines. It is similarly at the discretion of the local health authority/Medical Officer of Health to declare an outbreak over and to recommend when specific interventions may be halted. The end of a LTCF outbreak is generally considered when no new cases of ILI have appeared for $\geq 7$ days (22) after the onset of the last case (an interval equal to an average incubation period [three days] following the average period of communicability [three to five days]) or roughly two incubation periods.

\section{E. ANTIVIRAL USE DURING LTCF INFLUENZA OUTBREAKS}

It is generally accepted that antiviral drugs to treat and prevent influenza in residents, together with other infection control measures, are important for the control of influenza outbreaks in LTCF. Recommended practices with respect to antiviral use are as follows:

\section{Antiviral drug therapy}

i. Residents:

a. Oseltamivir or zanamivir treatment should be administered as soon as the clinical diagnosis of influenza has been made because residents of LTCF are, by definition, at increased risk of complications (6).

b. Nasal/nasopharyngeal secretions should be tested to confirm the diagnosis but treatment initiation should not wait for the test result.

c. Antiviral therapy works best when initiated within the first $48 \mathrm{~h}$ after symptom onset. However, these medications can still help even if begun more than $48 \mathrm{~h}$ after illness onset.

d. The dosage regimens for adults are oseltamivir $75 \mathrm{mg}$ PO BID or zanamivir two inhalations BID. The treatment dose for oral oseltamivir for infants (younger than one year) is $3 \mathrm{mg} / \mathrm{kg}$ twice a day. Given that in Canada, oseltamivir is currently not approved for use in infants younger than one year of age, the use of the drug for this age group should be done on case-by-case scenario basis. The treatment dosages for children one year and older vary by the weight of the child: $30 \mathrm{mg}$ twice a day for children who weigh $15 \mathrm{~kg}$ or less, $45 \mathrm{mg}$ twice a day for children who weigh more than $15 \mathrm{~kg}$ and up to $23 \mathrm{~kg}, 60 \mathrm{mg}$ twice a day for those who weigh more than $23 \mathrm{~kg}$ and up to $40 \mathrm{~kg}$, and $75 \mathrm{mg}$ twice a day for those who weigh more than $40 \mathrm{~kg}$. Clinicians are advised to consult an infectious diseases clinician or pharmacist for dosing of premature infants in the extremely unlikely that they are residents of a LCTF. Zanamivir is approved for children seven years of age and older (two inhalations BID).

e. The recommended duration of therapy is five days.

f. There is no need to reduce doses in patients with mild to moderate reduced renal function and drug-drug interactions are also not generally a concern (6).

ii. Staff:

a. Staff with ILI during an outbreak should be offered antiviral treatment and sent home until their symptoms have resolved.

b. Dose is per E1id, above.

\section{Antiviral drug prophylaxis}

\section{i. Residents:}

a. Upon diagnosis of an influenza outbreak, all residents becoming ill should be treated (vide supra) while all non-ill residents regardless of whether they have received the current seasonal vaccine should be started on chemoprophylaxis with either oseltamivir or zanamivir. b. Generally, it is preferable to wait to initiate chemoprophylaxis until laboratory confirmation has been received.

c. The prophylactic doses are oseltamivir $75 \mathrm{mg} \mathrm{PO}$ once daily or zanamivir two inhalations once daily for adults. The prophylactic dose for oral oseltamivir for infants three months to younger than one year of age is $3 \mathrm{mg} / \mathrm{kg}$ once daily. Prophylaxis is not recommended for infants younger than three months of age. The dosages for children one year of age and older vary by the weight of the child: $30 \mathrm{mg}$ once a day for children who weigh $15 \mathrm{~kg}$ or less, $45 \mathrm{mg}$ once a day for children who weigh more than $15 \mathrm{~kg}$ and up to $23 \mathrm{~kg}, 60 \mathrm{mg}$ once a day for those who weigh more than $23 \mathrm{~kg}$ and up to $40 \mathrm{~kg}$, and $75 \mathrm{mg}$ once a day for those who weigh more than $40 \mathrm{~kg}$. Zanamivir is approved for children seven years of age and older (two inhalations once daily).

d. Prophylaxis should be continued for 14 days minimum or until the outbreak has been declared over (see section D above).

\section{ii. Staff:}

a. In the context of significant antigenic drift and/or vaccine mismatch for which suboptimal VE may reasonably be anticipated, and in particular in relation to $\mathrm{H} 3 \mathrm{~N} 2$ viruses this season, it is recommended that staff who provide resident care or conduct activities where they may have the potential to acquire or transmit influenza (21) should also take prophylactic antiviral medication during the outbreak, regardless of whether they have received the current season's influenza vaccine. This is because, despite some vaccine protection anticipated, a substantial proportion of vaccinated individuals, including healthy working-age adults, are anticipated to remain susceptible to drifted $\mathrm{H} 3 \mathrm{~N} 2$ viruses.

b. Antiviral prophylaxis recommendations should be reinstituted whenever an outbreak is declared even for the same subtype and within the same setting and season. The maximum duration of continuous prophylaxis should be eight weeks, but outbreaks managed with antivirals should generally be terminated well within this period. In the unusual event that the outbreak is more prolonged, control measures should be reassessed in consultation with the local Medical Officer of Health and other experts.

c. Doses and durations should be as in section E2ic above.

\section{Continued surveillance for ILI during an outbreak:}

a. Residents or staff developing ILI while on prophylactic doses of an antiviral agent need to be considered for treatment doses if influenza is diagnosed or considered likely.

b. Residents or staff receiving chemoprophylaxis who develop ILI should be assessed and tested to determine the cause of their illness, which may be due to the current virus, another viral agent or an oseltamivir- (or zanamavir)-resistant influenza virus.

Expert consultation is suggested to address: the cause, organize testing for NI resistance, and to assess the possible need to possibly switch to another NI.

ACKNOWLEDGMENTS: The authors wish to acknowledge the contribution of Suzana Sabaiduc and Catharine Chambers to the summary of recent virus surveillance findings. The authors also acknowledge the critical review of the present document by the Public Health Agency of Canada and the Infectious Diseases and Immunization Committee of the Canadian Paediatric Society and its review and endorsement by the AMMI Canada Guidelines Committee. AMMI Canada would like to acknowledge the Public Health Agency of Canada who contributed by funding the translation of the guidelines. The authors also extend appreciation to Ms Angela Nelson for her excellent secretarial assistance.

DISCLOSURES: Dr Fred Y Aoki: Honoraria: Merck; Research: Biocryst Inc. Dr Upton D Allen: Research: Hoffmann La Roche Inc. Dr H Grant Stiver: Honoraria: Hoffman La Roche Inc; Advisory Board: Hoffman La Roche Inc. Dr Gerald A Evans: Research: Biocryst Inc. Dr Michel Laverdiere and Dr Danuta Skowronski: None. 


\section{REFERENCES}

1. Schanzer DL, Sevenhuysen C, Winchester B, Mersereau T. Estimating influenza deaths in Canada, 1992-2009. PLoS ONE 2013;8:e80481.

2. Thompson WW, Shay DK, Weintraub E, et al. Mortality associated with influenza and respiratory syncytial virus in the United States. JAMA 2003;289:179-86.

3. Thompson WW, Shay DK, Weintraub E, et al. Influenza-associated hospitalizations in the United States. JAMA 2004;292:1333-40.

4. Public Health Agency of Canada. FluWatch. <www.phac-aspc.gc.ca/ fluwatch/14-15/index-eng.php> (Accessed December 19, 2015).

5. World Health Organization. WHO recommendations on the composition of influenza virus vaccines. <www.who.int/influenza/ vaccines/virus/recommendations/en/index.html> (Accessed December 19, 2015).

6. Aoki FY, Allen UD, Stiver HG, Laverdière M, Evans GA. The use of antiviral drugs for influenza: A foundation document for practitioners. Can J Infect Dis Med Microbiol 2013;24(Suppl C):1C-15C.

7. Bush RM, Bender CA, Subbarao K, Cox NJ, Fitch WM. Predicting the evolution of human influenza A. Science 1999;286:1921-5.

8. Cox NJ, Bender CA. The molecular epidemiology of influenza viruses. Semin Virol 1995;6:359-70.

9. Ndifon W, Wingreen NS, Levin SA. Differential neutralization efficiency of hemagglutinin epitopes, antibody interference, and the design of influenza vaccines. Proc Natl Acad Sci USA 2009;106:8701-6.

10. Popova L, Smith K, West AH, et al. Immunodominance of antigenic site $B$ over site $A$ of hemagglutinin of recent $\mathrm{H} 3 \mathrm{~N} 2$ influenza viruses. PLoS One 2012;7:e41895.

11. Koel BF, Burke DF, Bestebroer TM, et al. Substitutions near the receptor binding site determine major antigenic change during influenza virus evolution. Science 2013;342:976-9.

12. Jin $H$, Zhou $H$, Liu $H$, et al. Two residues in the hemagglutinin of A/Fujian/411/02-like influenza viruses are responsible for antigenic drift from A/Panama/2007/99. Virology 2005;336:113-9.

13. Skowronski DM, Janjua NZ, De Serres G, et al. A sentinel platform to evaluate influenza vaccine effectiveness and new variant circulation, Canada 2010-11 season. Clin Infect Dis 2012;55:332-42.
14. Ohmit SE, Petrie JG, Malosh RE, et al. Influenza vaccine effectiveness in the community and the household. Clin Infect Dis 2013;56:1363-9.

15. Skowronski DM, Janjua NZ, De Serres G, et al. Low 2012-13 influenza vaccine effectiveness associated with mutation in the egg-adapted $\mathrm{H} 3 \mathrm{~N} 2$ vaccine strain not antigenic drift in circulating viruses. PLoS One 2014;9:e92153.

16. Andrews N, McMenamin J, Durnall H, et al. Effectiveness of trivalent seasonal influenza vaccine in preventing laboratoryconfirmed influenza in primary care in the United Kingdom: 2012/13 end of season results. Euro Surveillance 2014;19:5-13.

17. McLean HQ, Thompson MG, Sundaram ME, et al. Influenza vaccine effectiveness in the United States during 2012-13: Variable protection by age and virus type. J Infect Dis 2014 November 18 [Epub ahead of print]

18. European Centre for Disease Prevention and Control (ECDC). Surveillance report: Influenza virus characterization. Summary Europe, September 2014. <www.ecdc.europa.eu/en/publications/ Publications/Influenza-ERLI-Net-report-Sept-2014.pdf > (Accessed December 19, 2015).

19. European Centre for Disease Prevention and Control (ECDC). Surveillance report: Influenza virus characterization. Summary Europe, November 2014. <www.ecdc.europa.eu/en/publications/_ layouts/forms/Publication_DispForm.aspx?List=4f55ad51-4aed-4 3 32b960-af70113dbb90\&ID=1235> (Accessed December 19, 2015).

20. Castilla J, Martinez-Baz- I, Navascues A, et al. Vaccine effectiveness in preventing laboratory-confirmed influenza in Navarre, Spain: 2013/14 mid-season analysis. Euro Surveill. 2014;19:pii:20700.

21. Advisory Committee Statement - National Advisory Committee on Immunization (NACI). Statement on seasonal influenza vaccine for 2014-2015.

22. United States Centers for Disease Control \& Prevention. Interim guidance for influenza outbreak management in long-term care facilities (last updated December 19, 2011) <www.cdc.gov/flu/ professionals/infectioncontrol/ltc-facility-guidance.htm $>$ (Accessed December 19, 2015). 


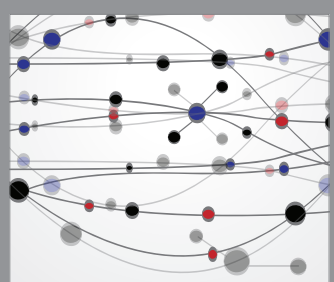

The Scientific World Journal
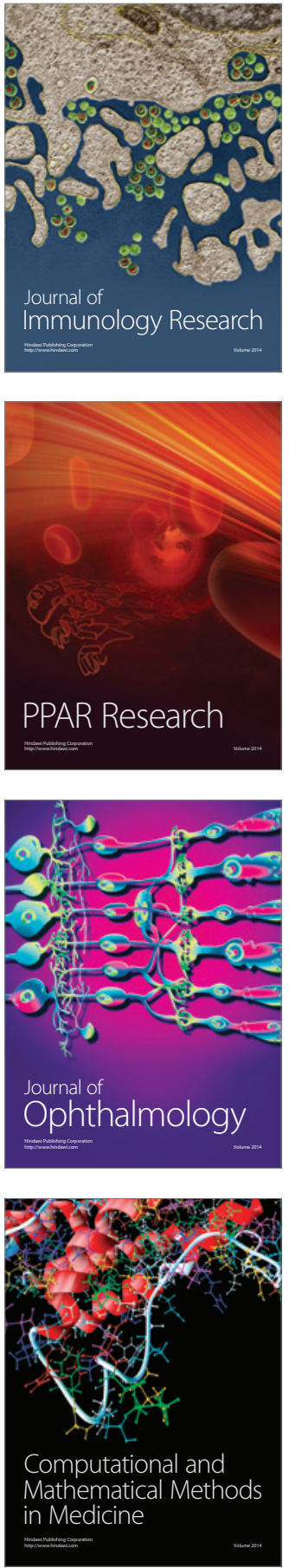

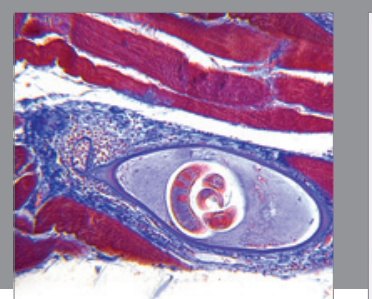

Gastroenterology Research and Practice

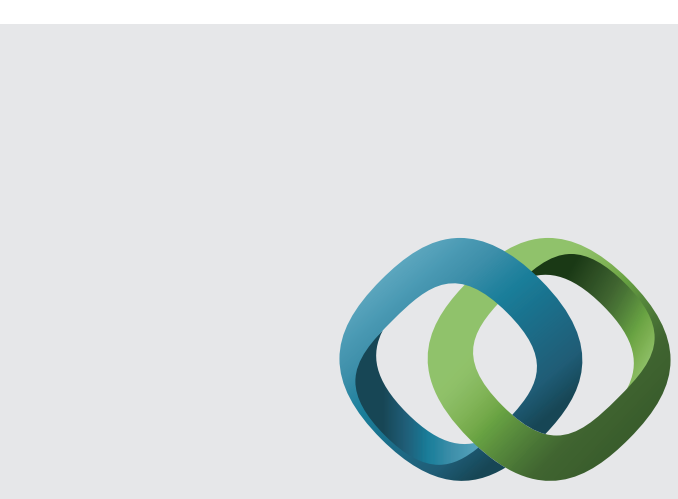

\section{Hindawi}

Submit your manuscripts at

http://www.hindawi.com
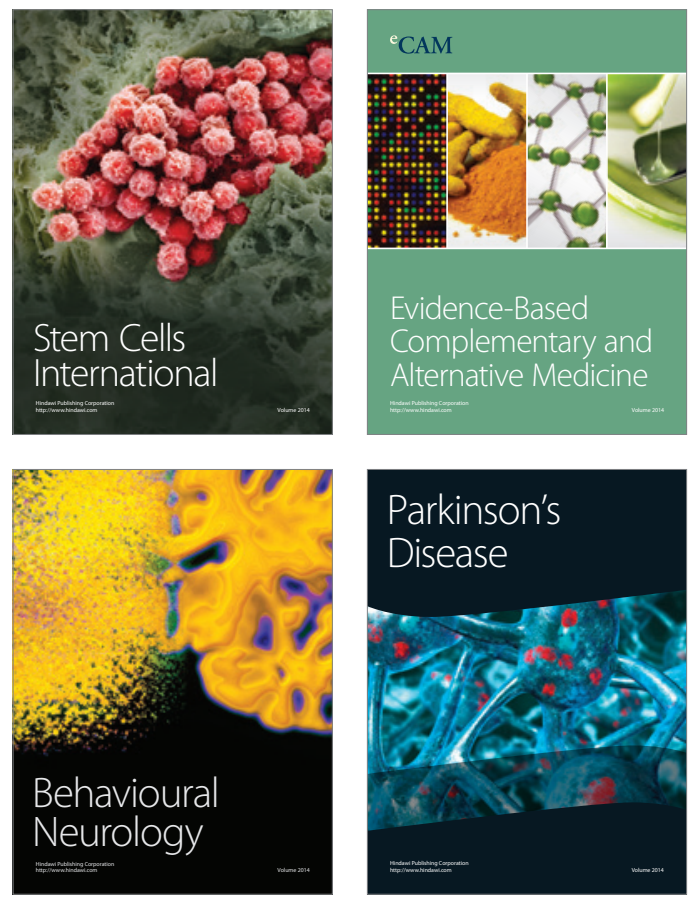
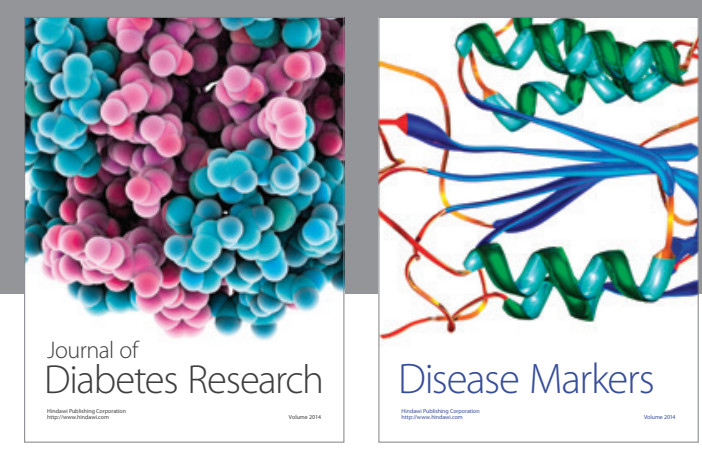

Disease Markers
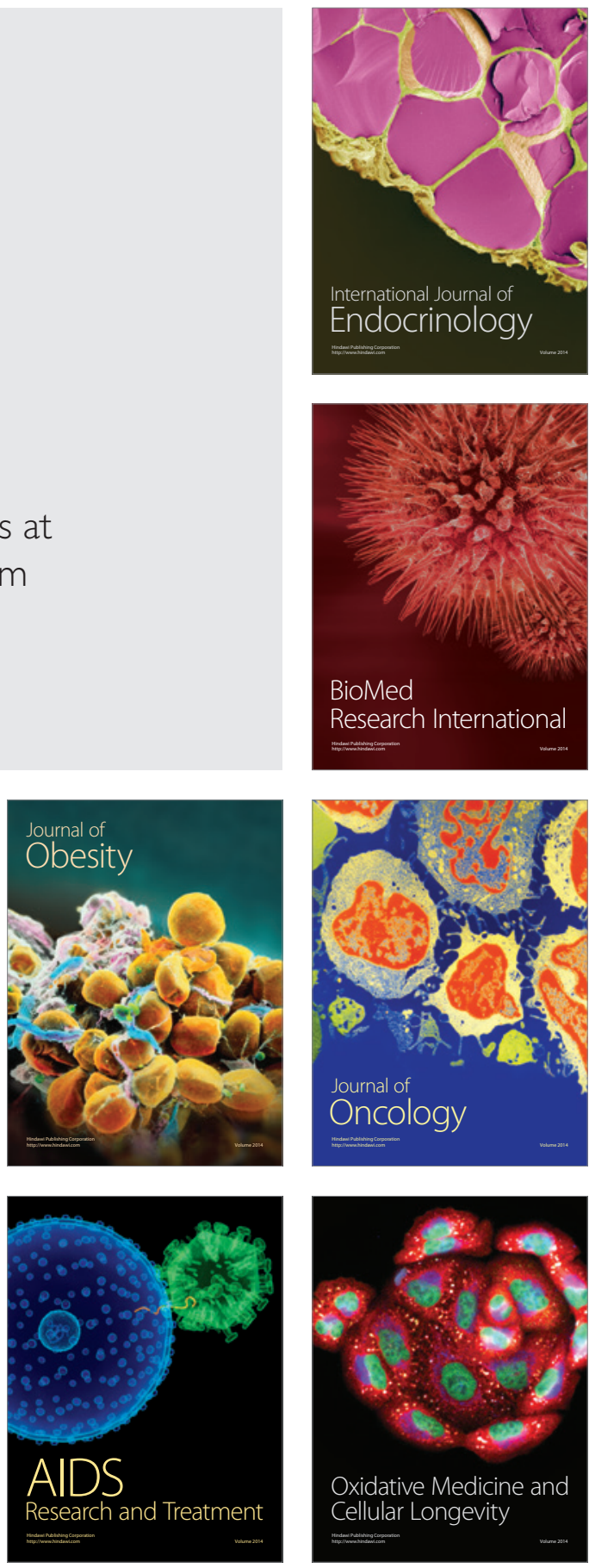